\title{
Consideration for Inhibiting Over-reliance of Autonomous Vehicle
}

\author{
Toshiya Arakawa, ${ }^{\text {a, }}$ \\ ${ }^{\mathrm{a}}$ Dept. of Mechanical Systems Engineering, Aichi University of Technology, Gamagori, Aichi 443-0047, Japan \\ *Corresponding Author: arakawa-toshiya@aut.ac.jp
}

\begin{abstract}
In order to realize level 3 of autonomous vehicles, human factors should be adequately considered with respect to driving autonomous vehicles. This paper verified the excessive reliance of drivers on an autonomous system or autonomous vehicle. From experiment, drivers tended to look at the areas that were unrelated to driving and had a foot attached on a breaking pedal in the case of autonomous driving scenario. However, the driver who was conscious of the risk of fault shown the opposite tendency. Hence, it was suggested that the way of providing information by HMI can control driver and driver's state and make driver prepare for the emergency of autonomous driving.
\end{abstract}

Keywords: autonomous vehicle, over-reliance, eye movement.

\section{Introduction}

Recently, there is a growing interest in developing autonomous vehicles by automakers and others. In 1939, at the New York World's fair General Motors (GM) hosted an exhibit called "Futurama." GM envisioned a future in which many autonomous vehicles would drive in a city with a lot of green areas. Hence, the idea of an autonomous vehicle is not recent. Several attempts were made to realize the idea of an autonomous vehicle. These included the development of autonomous vehicles such as Stanford Cart, Boss by Carnegie Mellon University, Shelly by Audi TTS, and Toyota Prius by Google. Additionally, an increasing number of automakers and others are also unveiling their plans. In December 2013, Ford unveiled a self-driving research car called a modified Fusion Hybrid. In 2017 in Gothenburg, Sweden, Volvo announced the introduction of 100 autonomous vehicles. Google has a fleet of fully autonomous Priuses and will release the corresponding technology in 2018. Nissan plans to announce the release of the first "commercially viable" self-driving system by 2020 . GM, Audi, BMW, Tesla and others also have autonomous vehicles under development. The 2014 Mercedes-Benz S-Class was the first car in the market that was fully capable of driving itself. With these developments, the idea of a fully autonomous vehicle and not just a partial autonomous vehicle like a vehicle equipped with ACC (adaptive cruise control) and/or LKAS (lane keeping assist system), may come true. An HIS automotive study predicted that there will be nearly 54 million self-driving cars in the world by 2035 . The study also predicted that after 2050, nearly all vehicles in use are likely to be self-driving cars or self-driving commercial vehicles. As an increasing number of autonomous vehicles are developed, the relation between the vehicle and driver will become more complex.

As the autonomous vehicle is a very feasible technology, people expect a mass production of autonomous vehicles. However, several problems need to be solved in order for autonomous vehicles to be produced. These problems include law revision, infrastructure development of P2P (Person to Person), V2P (Vehicle to Person), V2I (Vehicle to Infrastructure), and V2V (Vehicle to Vehicle) communication. Additionally, as the current focus of developers includes realizing level 3 of autonomous vehicles ${ }^{(1)}$, human factors should be adequately considered with respect to driving autonomous vehicles. A study indicated that drivers were incentivized and their mental workload (such as the effort in extending an arm) was reduced when ACC (Adaptive Cruise Control) was used. It also suggested that the drivers tend to rely heavily on the system ${ }^{(2)}$. However, the driver could also rely on system excessively ${ }^{(2)}$. Thus, it is very important to verify whether or not a driver relies excessively on the autonomous vehicle. The manner in which the driver handles a normal situation or relies on the autonomous vehicle excessively is also important. If the results reveal a 
tendency to excessively rely on autonomous vehicles, then this could be recognized as a negative effect of autonomous vehicles, and mechanisms to deal with the same can be investigated.

This study analyzes whether or not drivers excessively rely on autonomous vehicle excessively from the human factor viewpoint. This paper is divided as follows. The experimental situation and methods are introduced in Section 2. Section 3 discusses the results of the experiment. In section 4 , the analyzed results are demonstrated. Section 5 consists of the conclusions.

\section{Experiments}

Three subjects (aged 20-21 years) were involved in the experiment. A driving simulator (D3sim, by Mitsubishi precision Co., Ltd.) was used to measure the effect of human factors on autonomous driving. Drivers were instructed to drive on the urban-area course for approximately fifteen minutes by controlling the handle, accelerator pedal and braking pedal on their own. This was the manual-driving scenario. After a break of approximately $15 \mathrm{~min}$, the drivers were instructed to drive on the urban-area course for another period of approximately 15 min. However, in this session, the handle, accelerator pedal and braking pedal were automatically controlled. Thus, the drivers did not have to control the vehicle themselves. This was the autonomous driving scenario. Drivers were also asked to press the brake pedal when a presence of the collision risk was determined, and/or the system fault occurred suddenly, and/or the drivers could not control the driving simulator. It should be noted that in reality, no system fault occurred. However, in the autonomous driving scenario, an event where a man suddenly rushed out on the course after approximately 6 min of driving was included.

In both the manual and autonomous driving scenarios, the driver's eye movement, facial expression, braking behavior and experimental situation were recorded and measured. The driver's eye movements were recorded and measured by an eye-mark recorder (T.K.K.2950 TalkEye Lite, by Takei Scientific Instruments Co., Ltd.). The driver's facial expression and braking behavior were recorded as motion pictures by a CCD camera and a small infrared camera, respectively. The experimental situation was recorded by a video camera (Everio, by JVC). These scenes were combined into a single screen by a split screen unit (Figure 1). The timing of attaching a foot on the braking pedal or hitting the brakes was judged by the

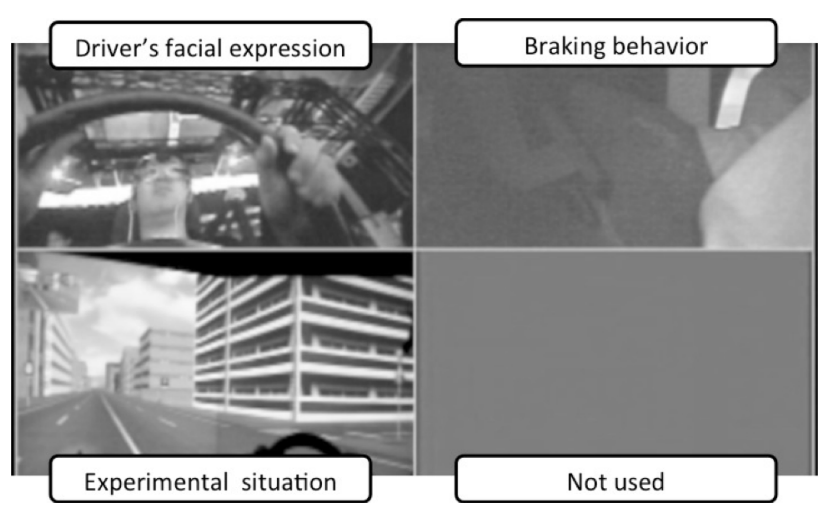

Fig.1 Driver's behavior. Upper left: driver's facial expression, upper light: braking behavior, lower left: experimental situation.

experimenter from the motion picture of driver's braking behavior.

\section{Results}

The heat map (that is, time-series data and probability of eye-movement that indicates where a driver tends to gaze), the time-series graph of autonomous braking, the driver's brake work were illustrated in Fig. 2 and Fig.3 for driver A, B, and C, respectively. The heat map shows where and how long the driver gazed in the driving direction. The horizontal direction was the angle of the gazing direction, wherein the center was $0^{\circ}$, the left end of the field of vision was $-90^{\circ}$, and the right end of the field of vision was $90^{\circ}$. The vertical direction indicated the driving time from the start of the vehicle. The heat map illustrated the probability density of the gazing point in a time series. If an area in heat map was white colored at a point, it implied that the probability of the driver's gaze in the area was relatively high. Conversely, if an area in the heat map was black colored at a point, this indicated that the probability of the driver's gaze in the area was relatively low.

In the time-series graph of autonomous braking and the driver's brake work, the gray line depicted the timing of autonomous braking, and the dark gray line represented the timing of attaching a foot on the braking pedal or hitting the brakes.

\section{Discussion}

Figure 2 indicated that driver A and B mainly looked in the driving direction, and they appeared to concentrate on the safety of driving in the case of the manual driving scenario. This figure indicated that drivers tended to look in 

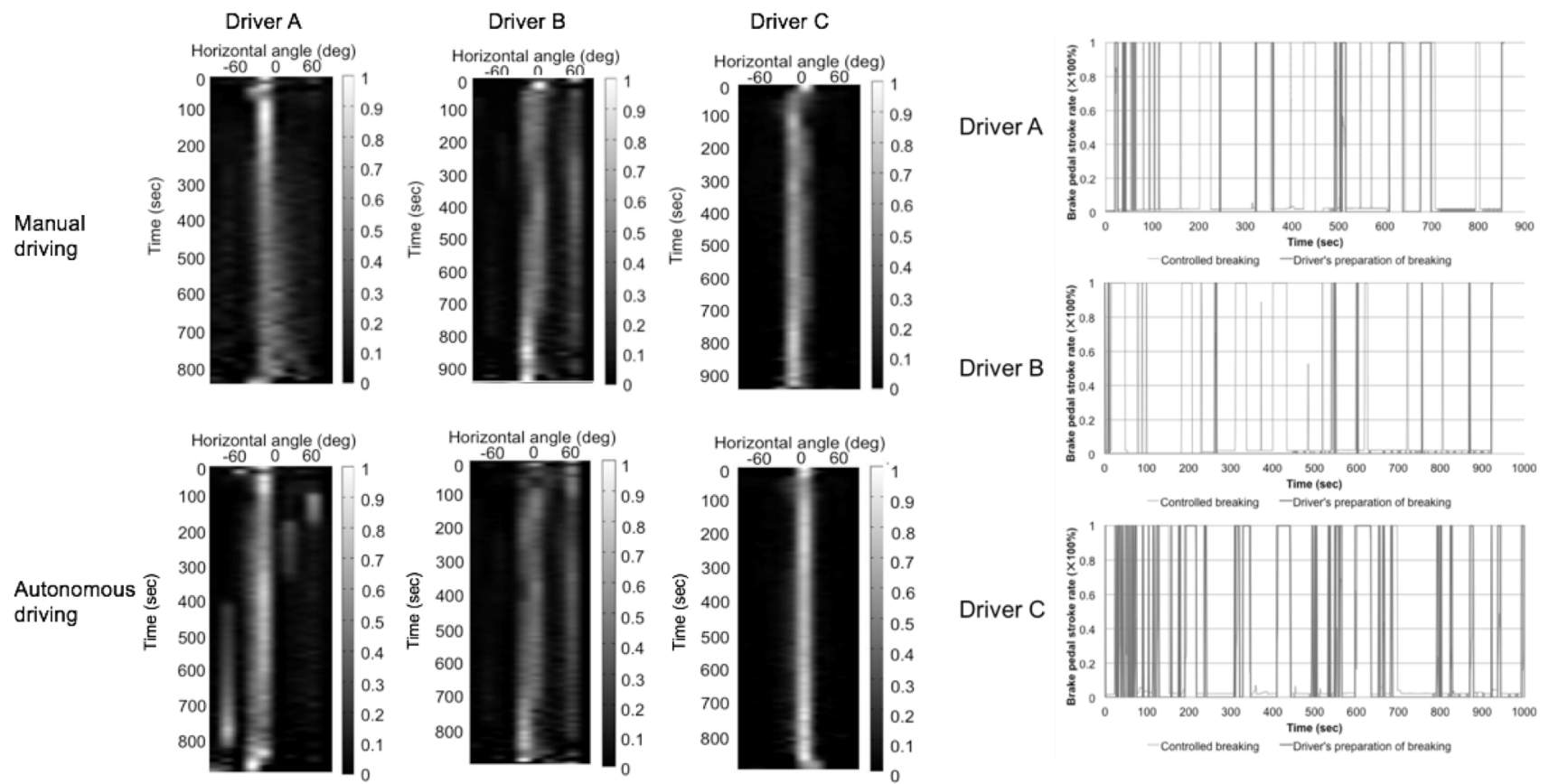

Fig.2 Eye movement of each driver on manual driving scenario and autonomous driving scenario.

Fig.3 Braking behavior of each driver on manual driving scenario and autonomous driving scenario.

the driving direction, but they also looked at the areas that were unrelated to driving in the case of automated driving scenario. For example, the driver A tended to look in the area between $-80^{\circ}$ and $-60^{\circ}$ with time. Driver B tended to look in the right from the center, and the frequency of looking at the $60^{\circ}$ area increased. This suggested that driver $\mathrm{A}$ and $\mathrm{B}$ were slightly nervous about driving in the manual-driving scenario, but their mental workloads were decreased. Thus, they could look not only in the driving direction but also in the areas that were unrelated to driving. This tendency was caused by a reliance on the autonomous system. If the drivers did not over rely on the autonomous system, they looked only in the driving direction. Generally speaking, drivers get to look at the areas that were unrelated to driving only after spending a long time driving ${ }^{(3)}$. However, in this study, the subjects looked at the areas that were unrelated to driving as soon as they started driving. Hence, it could be concluded that the subjects relied heavily on autonomous driving.

From figure 3, drivers tended to not attach a foot on the braking pedal with time. If drivers did not rely on the autonomous vehicle, they attached a foot on the braking pedal constantly, and hit the brake every time that the autonomous braking was in progress. The subjects in the study rarely placed a foot on the braking pedal. This suggested that they seemed to rely on the autonomous vehicl

e. Driver A and B did not attach a foot to the braking pedal, and were therefore not prepared for the scenario when a man rushed out. The drivers assumed that the autonomous system would avoid the obstacles, and would always stop the vehicle before it collided with the obstacles. Thus, the drivers relied on the autonomous driving to the extent that they were not prepared by attaching a foot on the braking pedal, in spite of being warned about the possibility of system failure.

It was observed from the heat map and the braking behavior that driver A relied too heavily on the autonomous vehicle. Conversely, from figure 2 it was revealed that driver $\mathrm{C}$ looked in the driving direction in both the manual driving and the autonomous driving scenarios, and $\mathrm{s} /$ he did not display a tendency to look at the areas that were unrelated to driving. Although driver $\mathrm{C}$ had a license, the driver rarely drove. This limited his/her driving experience. Additionally, the subject was afraid that the system fault might occur suddenly. Thus, the subject concentrated on driving excessively. Hence, taking these factors into account, the subject's mental workload was very high and $\mathrm{s} /$ he did not look at the areas that were unrelated to driving. In fact, after the subject completed the driving task, s/he revealed that $\mathrm{s} /$ he concentrated on driving because of the fear that the system could fail. This was supported by the 
part of driver $\mathrm{C}$ in figure 3 . In the part of driver $\mathrm{C}$ in figure 3 , almost all the gray lines and the dark gray lines overlapped, suggesting that the subject did not rely on autonomous driving, and that s/he had a foot attached on a braking pedal throughout the action.

We considered the reason that driver $\mathrm{C}$ did not display a tendency to look at the areas that were unrelated to driving and had a foot attached on a breaking pedal in the case of autonomous driving scenario. After experiment, driver $\mathrm{C}$ told that $\mathrm{s} /$ he believed that the system fault might occur suddenly and s/he were nervous in order to drive safely. Thus, it was found that the way of instruction controls the driver's state and make driver inhibit over-reliance on the autonomous driving. Hence, it was suggested that the way of providing information by HMI can control driver and driver's state and make driver prepare for the emergency of autonomous driving.

\section{Conclusion}

This paper verified the excessive reliance of drivers on an autonomous system or autonomous vehicle. From the eye-movement viewpoint, drivers gazed at objects unrelated to driving, such as side strips and roadside trees. Additionally, drivers tended not to attach a foot on the braking pedal as they relied on the autonomous system, and believed that the autonomous vehicle would stop and not hit the obstacle. Thus, the mental workload of driver in the autonomous system driving scenario was less than the mental workload of the driver in the manual driving scenario.

\section{Acknowledgment}

This research was supported by KAKENHI (Grant-in-Aid for Scientific Research) 15K21486.

\section{References}

(1) Frank, D. (2014): "Autonomous Vehicles: The Automotive Ecosystem", https://frankdiana.wordpress.com/2014/03/03/autonom ous-vehicles-the-automotive-ecosystem/, Accessed 22 January 2016.

(2) Inagaki, T: "Human's Overtrust in and Overreliance on Driver Assistance Systems", ICICE Technical Report SSI2010-6, pp.21-24, 2010
(3) Arakawa, T., Matsuo, N. and Kinoshita, M.: "Trial Evaluation on Drivers' Unfocused Attention using Gaze Analysis", Review of Automotive Engineering, Vol.27, No.2, pp.357-359. 2006 